\title{
CAPACITY IMPROVEMENT OF MIMO-OFDMA SYSTEM USING ADAPTIVE RESOURCE ALLOCATION ALGORITHM
}

\author{
Thazin Myat ${ }^{1}$, Zaw Min Naing², Thiri Thandar Aung 3 , Thet Paing Phyo ${ }^{4}$ \\ ${ }^{I}$ Ph. D. Candidate, EC Department, YTU, Yangon, Myanmar \\ ${ }^{2}$ Pro-Rector, EC Department, TU (Maubin), Yangon, Myanmar \\ ${ }^{3}$ Lecturer, EC Department, YTU, Yangon, Myanmar \\ ${ }^{4}$ Associate Professor, EC Department, YTU, Yangon, Myanmar
}

\begin{abstract}
The user demands for wireless communication are increasing but the existing networks are not able to fulfill the high data rate of future communication services. More bandwidth can support high data rate wireless access. Due to spectral limitations, it is very expensive to increase. Therefore, the available bandwidth is needed to use efficiently. Orthogonal Frequency Division Multiplexing (OFDM) has been explored as a promising air interface technology to utilize the bandwidth effectively. OFDM can be exploited as a multiple access technique, called Orthogonal Frequency Division Multiple Access (OFDMA) that can support both frequency and multiuser diversity. In order to serve the user population, one of the most sophisticated techniques, resource allocation technique, can be integrated into the OFDMA system. Multiple Input Multiple Output (MIMO) technology can increase the spectral efficiency because multiple data streams can be simultaneously transmitted over the channel. The combination of MIMO technology and OFDMA system can achieve both higher spectral efficiency and better Quality of Service (QoS) such as capacity, fairness, and Bit Error Rate (BER). In this paper, a MIMO-OFDMA system is developed for downlink transmission within one single cell. Two optimization methods such as subcarrier allocation and power allocation are used for resource allocation. To decompose the MIMO channel into parallel single channels, Eigen Value Decomposition (EVD) is used. Subcarrier selection is based on the priority of the user by using the dominant eigen channels. Water-filling algorithm is adapted for power distribution among users. The aim is to maximize the system capacity subject to constraints on total power and proportional fairness. The performance of the system is evaluated by comparing the static allocation method (equal subcarrier allocation with equal power) and two adaptive allocation methods (adaptive subcarrier allocation with equal power and adaptive subcarrier allocation with adaptive power).
\end{abstract}

Keywords: MIMO-OFDMA System, Resource Allocation Algorithm, System Capacity, Water-filling Algorithm.

\section{INTRODUCTION}

Orthogonal Frequency Division Multiplexing (OFDM) is a promising technique to fulfill the ability to combat multipath fading, shadowing, Inter Symbol Interference (ISI) and time-varying injurious effects in wireless environment. OFDM is often used as multiple access technique such as Orthogonal Frequency Division Multiple Access (OFDMA) that can give both the frequency diversity and the multiuser diversity to improve the system efficiency. The application of Multiple Input Multiple Output (MIMO) system can greatly improve the performance of the physical layer in the wireless communication systems. By employing multiple antennas at both the transmitter and the receiver, multiple spatial channels can be created to spatially multiplex the multiple streams simultaneously. Two popular techniques in MIMO system are spatial diversity that can support link reliability and spatial multiplexing that can support the high data rate. MIMO technology offers the significant improvement in the data throughput and link reliability without additional bandwidth or power. Because of these properties, MIMO system is an essential part of the modern communication standards such as IEEE 802.11n (Wi-Fi), Long Term Evolution (LTE), WiMAX and $\mathrm{HSPA}^{+}$[1].
The combination of MIMO and OFDMA can handle the problems caused by multipath channel more efficiently to achieve a high performance transmission in advanced wireless networks [2] and [3]. Resources can be considered as a three dimension such as subcarriers, time slots and spatial layers in frequency, time and space domain respectively [4]. Resource allocation is allocating the resources to the users for their various services from the Base Station (BS). Optimization problems of resource allocation aim either maximizing the total system capacity under power constraints, defined as Rate Adaptive (RA) [513] or minimizing the total power under rate constraints called Margin Adaptive (MA) [14-16]. To solve these optimization problems, there are two allocation methods: fixed and adaptive allocation. Time Division Multiple Access (TDMA) and Frequency Division Multiple Access (FDMA) are used as multi-access schemes to allocate the predetermined time slot or frequency for each user. For fixed allocation, channel condition is neglected. These two fixed allocation schemes are compared in [17]. These methods degrade the performance of the system because the gap of channel gain causes high error probability in the MIMO system. And the most of the resources might be wasted. 
On the other hand, adaptive allocation allocates the resources to the users according to the channel conditions. Therefore, the usage of resources will be more effective and full use of the multiuser diversity to achieve higher performance. In this paper, MIMO-OFDMA system is firstly modeled by using spatial multiplexing because it is concentrated on the system capacity. And a resource allocation algorithm is developed and combined with MIMO-OFDMA system. In this algorithm, three methods such as static allocation (equal subcarrier with equal power) and two adaptive allocation methods (adaptive subcarrier with equal power and adaptive subcarrier with adaptive power) are developed and compared. The whole system is simulated by MATLAB simulation tool.

\section{SYSTEM MODEL}

In the downlink point to multipoint infrastructure, there are two parts: base station (transmitter) and users (receivers) with heterogeneous service requirements as shown in Figure 1. The base station (BS) must use base station transmitter and each user must use user $\mathrm{k}$ receiver. In this system, the channel state information (CSI) from all users is sent to resource allocation algorithm at the BS. It is supposed that the perfect CSI is known at both the transmitter and the receiver. At the BS, the algorithm allocates resources such as frequency, power and antennas for each user by using this CSI. It is also assumed that this resource allocation is sent to each user via a separate channel. The transmitter transmits the user's data using this resource allocation to each user via MIMO channel. At the receiver, the user recovers the data with the help of resource allocation scheme received from the transmitter. The resource allocation scheme is updated as fast as the CSI is changed.

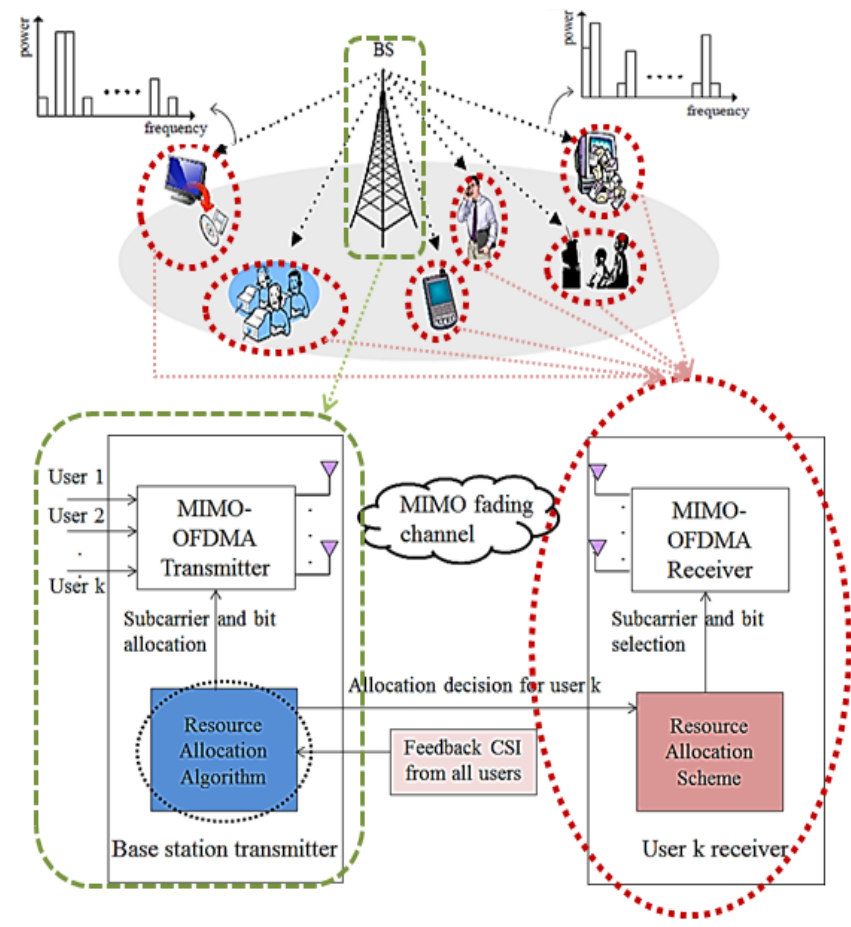

Fig -1: System model on downlink point to multipoint (PMP) network platform
The following suppositions are used in this paper:

- It is assumed that time-varying channels between transmitter and the users as frequency selective Rayleigh fading.

- This channel can be held as constant within allocation period.

- $\quad$ Only one user can use each subcarrier at each time.

- $\quad$ Each user experiences flat fading on each subcarrier because ISI is completely removed by OFDM technique.

- The receiver is known the perfect CSI and feedbacks to BS correctly.

In this paper, a MIMO-OFDMA system consists of a total of $\mathrm{U}$ users sharing $\mathrm{S}$ subcarriers in a single cell. The channel gain of user ' $u$ ' on subcarrier's' is defined as $g_{u, s}$. The total power is $\mathrm{P}_{\text {tot }}$ and the power of user ' $\mathrm{u}$ ' on subcarrier ' $\mathrm{s}$ ' is denoted as $p_{u, s}$. The available bandwidth is $\mathrm{B}$. The noise power spectral density is $\mathrm{N}_{0}$. The ' $\mathrm{u}$ ' user's received SNR is

$\operatorname{SNR}=\frac{\mathrm{p}_{\mathrm{u}, \mathrm{s}}\left|\mathrm{g}_{\mathrm{u}, \mathrm{B}}^{(\mathrm{i})}\right|^{2}}{\mathrm{~N}_{0} \frac{\mathrm{B}}{\mathrm{S}}}$

The total data rate of user ' $u$ ' is

$\mathrm{R}_{\mathrm{u}}=\frac{\mathrm{B}}{\mathrm{S}} \sum_{\mathrm{i}=1}^{\mathrm{M}} \log _{2}(1+\mathrm{SNR})$

To perform channel adaption in MIMO, the MIMO channel is decomposed into a number of parallel (non-interfering) single channels by using Eigen Value Decomposition (EVD). Each subcarrier has narrowband channel with 'Tx' antennas at the base station and ' $R x$ ' antennas at the receiver. It can be modelled by an 'Rx' $x$ 'Tx' channel matrix $\mathrm{H}=\left[\mathrm{h}_{\mathrm{ij}}\right]$, where $\mathrm{h}_{\mathrm{ij}}$ is the channel gain at the receive antenna $\mathrm{i}$ from the transmit antenna $\mathrm{j}$ as shown in Figure 2.

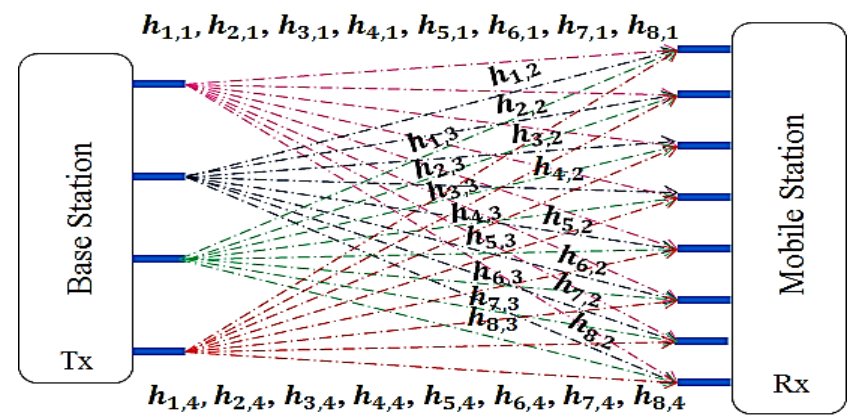

Fig -2: Channel decomposition of $4 \times 8$ antenna system for one user

The Hermitian $\mathrm{H}^{\mathrm{H}} \mathrm{H}$ can be decomposed by using EVD as follows

$\mathrm{H}^{\mathrm{H}} \mathrm{H}=\mathrm{VDV}^{\mathrm{H}}$

Where $\mathrm{D}=\operatorname{diag}\left\{\lambda_{1}, \lambda_{2}, \quad \lambda_{3}, \ldots, \lambda_{\mathrm{M}}\right\}, \quad$ where $M \leq \min \left\{N_{R}, N_{T}\right\} \cdot \lambda_{m}$ are the eigenvalues and $m \in[1, M]$ is the spatial index. According to the EVD, $\mathrm{V}$ is the unitary 
eigenvector matrix of $\mathrm{H}^{\mathrm{H}} \mathrm{H}$. Each channel carries a portion of user's data. The optimization problem is

$$
\max _{\mathrm{p}_{\mathrm{u}, \mathrm{s}}} \frac{\mathrm{B}}{\mathrm{s}} \sum_{\mathrm{u}=1}^{\mathrm{U}} \sum_{\mathrm{s} \in \Omega_{\mathrm{u}}}\left[\sum_{\mathrm{i}=1}^{\mathrm{M}} \log _{2}\left(1+\frac{\mathrm{p}_{\mathrm{u}, \mathrm{s}}\left|\mathrm{g}_{\mathrm{u}, \mathrm{s}}^{(\mathrm{i})}\right|^{2}}{\mathrm{~N}_{0} \frac{\mathrm{B}}{\mathrm{s}}}\right)\right]
$$

Subject to the following three constraints:

$\mathrm{C} 1$ : Each subchannel is apportioned to only one user,

$$
\bigcup_{\mathrm{u}=1}^{\mathrm{U}} \Omega_{\mathrm{u}} \underline{\mathrm{C}}\{1,2, \ldots, \mathrm{S}\} \text { with }\left\{\Omega_{\mathrm{u}}\right\}_{\mathrm{u}=1}^{\mathrm{U}} \text { being disjoint sets, }
$$

C2: The sum of power allocating for all subcarriers is less than or equal to the total power,

$$
\sum_{\mathrm{u}=1}^{\mathrm{U}} \sum_{\mathrm{s} \in \Omega_{\mathrm{u}}} \mathrm{p}_{\mathrm{u}, \mathrm{s}} \leq \text { Ptot with } \mathrm{p}_{\mathrm{u}, \mathrm{s}} \geq 0, \forall \mathrm{u}, \mathrm{s} \text { and }
$$

C3: Proportional fairness constraints among users, $\mathrm{R}_{1}: \mathrm{R}_{2}: \ldots \ldots: \mathrm{R}_{\mathrm{U}}=\alpha_{1}: \alpha_{2}: \ldots \ldots: \alpha_{\mathrm{U}}$

where $\Omega_{\mathrm{u}}$ is the set of subcarriers assigned to user ' $u$ ' and the predetermined values $\{\alpha\}_{\mathrm{u}=1}^{\mathrm{U}}$ are used to ensure proportionality among users. Three constraints are denoted as $\mathrm{C} 1-\mathrm{C} 3$. To solve this optimization problem, equal or adaptive subcarrier allocation, bit allocation, and equal or adaptive power allocation schemes are proposed in this paper.

\section{MIMO-OFDMA SYSTEM}

In this proposed system, there are one transmitter (BS) and many receivers (users). Figure 3 shows a transceiver for one user. Transmitter is the BS. Receiver is for this user. There is only one $\mathrm{BS}$ and there may be more than one user at the receiver. Figure 4 expresses the process of MIMO-OFDMA software implementation. Transmitter is composed of user bit generation, resource allocation algorithm, symbol mapping, subcarrier and bit allocation, space time coding, and pilot insertion. According to the number of users, random bit is generated for each user by taking into account the space time coding rate. By generating these binary data, each user's data is achieved. To assign the subcarrier and power for each user, the perfect CSI is needed to create. It is assumed that this CSI is available at both the transmitter and the receiver.

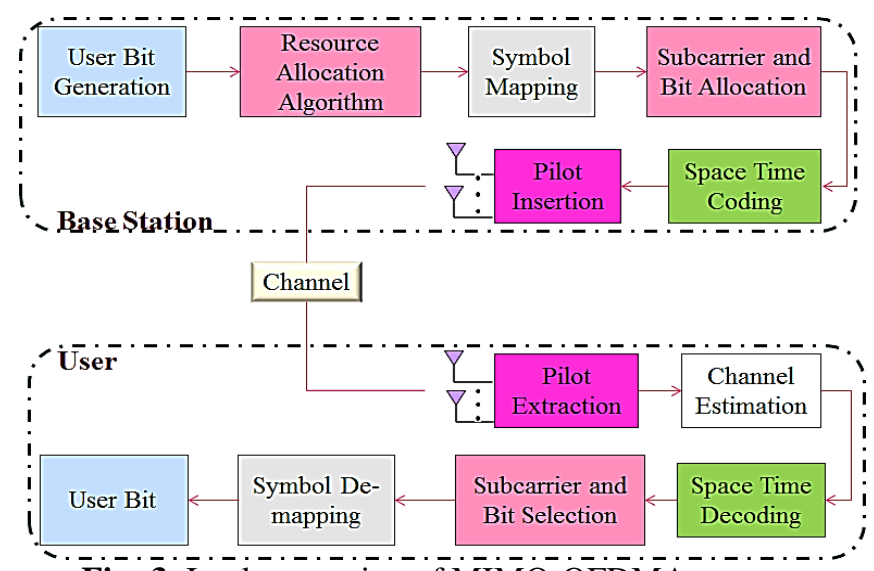

Fig -3: Implementation of MIMO-OFDMA system
Depending on the CSI, resource allocation scheme is developed. This scheme assigns the subcarriers or frequency, and power to each user. It is also assumed that this scheme is known at the receiver. In symbol mapping, each user's binary data is modulated by using modulation technique. These mapped data is allocated to the subcarriers assigned for each user.

Space time coding is used to reduce multipath propagation effects. There are two techniques: spatial diversity and spatial multiplexing. In this system, spatial multiplexing technique is used because system capacity is emphasized. In spatial multiplexing techniques, Vertical Bell Labs Layered Space-Time (V-BLAST) coding scheme is used. The assigned data is allocated to the transmit antennas by using this coding scheme. And the known pilots are added to the transmit data to estimate the channel. The different symbols are simultaneously transmitted over different antennas at the same time.

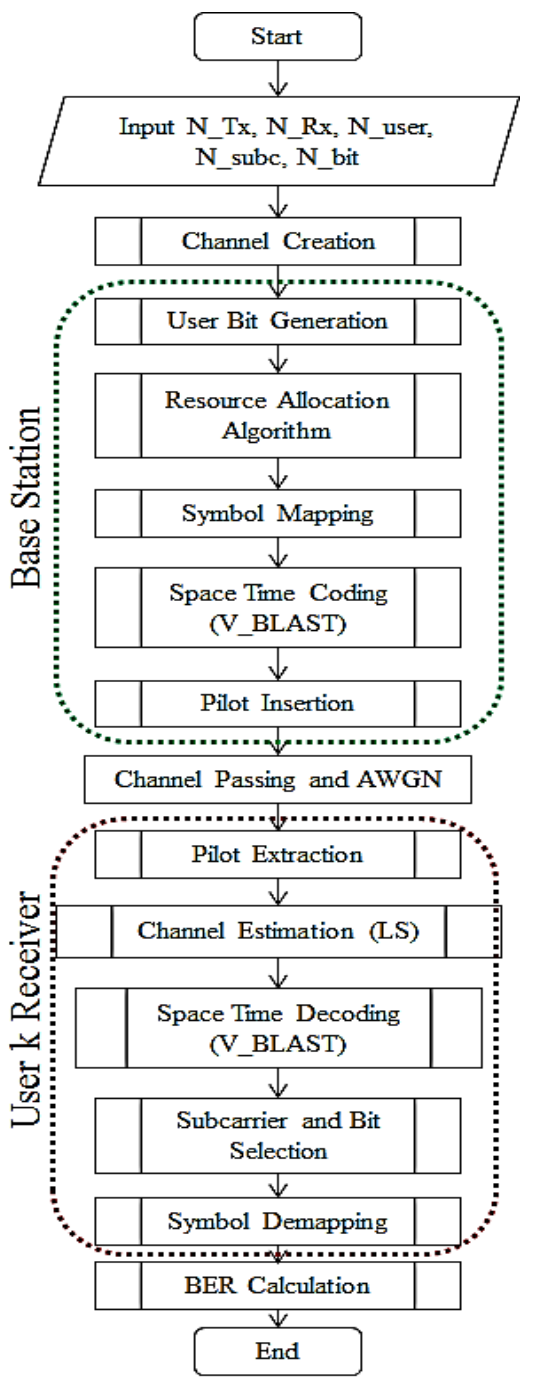

Fig -4: Software implementation of MIMO-OFDMA system

The data transmitted through the created channel is received by the users. At the receiver, the known pilots are extracted from the received data to estimate the channel. There are Zero Forcing (ZF) and Minimum Mean Square Error 
(MMSE) signal detection methods recovering the transmitted signal in the V-BLAST coding scheme. To get the transmitted signal, the channel is needed to estimate. In order to estimate the channel, training-based channel estimation technique, Least Square (LS) method is used. By using this estimated channel, the symbols are detected by using $\mathrm{ZF}$ detection method. For multiuser condition, the users' symbols are mixed with each other. Therefore, the user must use the known resource allocation decision to select the data on the user's assigned subcarriers. These data or symbols are de-mapped to get the user bit or binary data by using the modulation technique that is used by the mapper at the transmitter. This process is implemented and simulated by using MATLAB software tool.

\section{CHANNEL CREATION BY JAKES MODEL}

The performance of wireless communication systems is mainly governed by the wireless channel environment. Depending on the mobile speed, time variation of channel gain is governed by Doppler spectrum, which determines the time-domain correlation in the channel gain. To model the time-correlated channel variation as the mobile terminal moves, Jakes model is used.

It is assumed that all rays of the scattered components arriving in the uniform directions are approximated by $\mathrm{N}$ plane waves. Let $\theta_{\mathrm{n}}$ denote an angle of arrival for the nth plane wave, which is modeled as $\theta_{\mathrm{n}}=2 \pi \mathrm{n} / \mathrm{N}, \mathrm{n}=$ $1,2, \ldots, \mathrm{N}_{0} . \mathrm{N}_{0}$ is defined as 16 for $\mathrm{N}=2 \times\left(2 \times \mathrm{N}_{0}+1\right)$. A sum of $\mathrm{N}_{0}$ complex oscillator outputs with the frequencies of $\mathrm{w}_{\mathrm{n}}=\mathrm{w}_{\mathrm{d}} \cos \theta_{\mathrm{n}}, \mathrm{n}=1,2, \ldots, \mathrm{N}_{0}$, each corresponding to different Doppler shifts, is added to the output of a complex oscillator with a frequency of $\mathrm{w}_{\mathrm{d}}=2 \pi \mathrm{f}_{\mathrm{m}}$. The real $\mathrm{h}_{\mathrm{I}}(\mathrm{t})$ and imaginary $h_{Q}(t)$ parts in the total sum of the complex oscillators can be represented respectively as

$$
h_{I}(t)=2 \sum_{n=1}^{N_{0}}\left(\cos \emptyset_{n} \cos _{n} t\right)+\sqrt{2} \cos \emptyset_{N} \cos _{d} t
$$

And

$$
\mathrm{h}_{\mathrm{Q}}(\mathrm{t})=2 \sum_{\mathrm{n}=1}^{\mathrm{N}_{0}}\left(\sin \emptyset_{\mathrm{n}} \cos \mathrm{w}_{\mathrm{n}} \mathrm{t}\right)+\sqrt{2} \sin \emptyset_{N} \cos \mathrm{w}_{\mathrm{d}} \mathrm{t}
$$

Where $\emptyset_{\mathrm{n}}$ and $\emptyset_{\mathrm{N}}$ are the initial phases of the n-th Doppler shifted sinusoid and the maximum Doppler frequency $f_{m}$, respectively. The initial phase can be set to

$$
\begin{gathered}
\emptyset_{\mathrm{N}}=0 \\
\emptyset_{\mathrm{n}}=\pi \mathrm{n} /\left(\mathrm{N}_{0}+1\right), \mathrm{n}=1,2, \ldots, \mathrm{N}_{0}
\end{gathered}
$$

The complex output of the Jakes model can be represented as

$$
h(t)=\frac{E_{0}}{\sqrt{2 N_{0}+1}}\left\{h_{I}(t)+j h_{Q}(t)\right\}
$$

Where $E_{0}$ is the average of the fading channel [18].

\section{RADIO RESOURCE ALLOCATION SCHEME}

In this scheme, resources such as sub carriers or frequency and power are allocated to the best users based on the subchannel gains. To reduce complexity, this scheme is separated into two sections such as subcarrier allocation and power allocation. Static allocation is allocating the equal number of data subcarriers and equal power to each user. Therefore, adaptive subcarrier allocation and power distribution among users are described as follows:

\subsection{Adaptive Subcarrier Allocation}

Firstly, the parameters for this allocation are initialized. By using EVD, lamda $(\lambda)$ is calculated from all channels of each subcarrier. For each user, dominant eigen channels are achieved for all subcarriers by using eigen function. From channels of all subcarriers, the maximum eigen value is chosen as a subcarrier allocated for this user. This step 2 is allocating a subcarrier which is the most dominant eigen channel (the best channel) to each user. In step 3, the user with the least ratio of instantaneously achieved rate to its required proportion is firstly determined to assign the subcarriers. This is called priority-based policy. The remaining subcarriers are apportioned to the user with the best channel gain in which each user can get at most one unassigned subcarrier. Figure 5 demonstrates the process of adaptive subcarrier allocation with equal power distribution such that $\mathrm{p}_{\mathrm{u}, \mathrm{s}}$ is the power allocated to user ' $\mathrm{u}$ ' on subcarrier 's'.

$\mathrm{p}_{\mathrm{u}, \mathrm{s}}=\frac{\mathrm{P}_{\mathrm{tot}}}{\mathrm{S}}$

Where $\mathrm{P}_{\text {tot }}$ is the total transmitting power by the BS

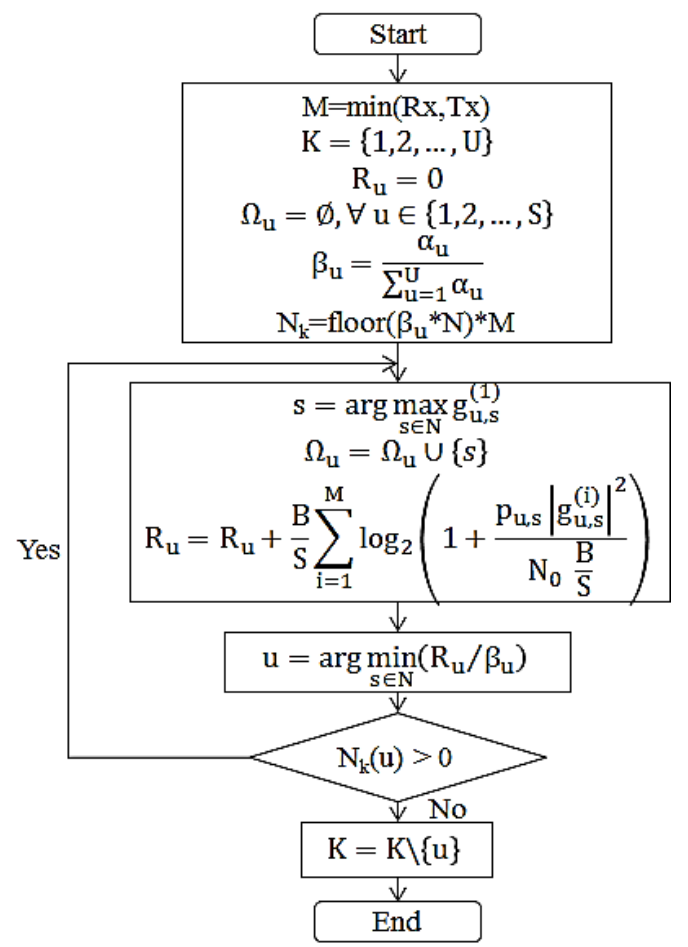

Fig -5: Flowchart of adaptive subcarrier allocation 
By allocating the subcarriers for each user adaptively, each user achieves the set of subcarriers which can be adjacent or non-adjacent according to the channel gains.

\subsection{Adaptive Power Allocation}

Power allocation is another technique to maximize the throughput of wireless communication systems. The process allocates different amounts of transmit power to each transmit antenna. The optimization problem in Equation 4 is formulated as

$\sum_{\mathrm{u}=1}^{\mathrm{U}} \sum_{\mathrm{s} \in \Omega_{\mathrm{u}}} \frac{1}{\mathrm{~S}} \log _{2}\left(1+\frac{\mathrm{p}_{\mathrm{u}, \mathrm{s}}\left|\mathrm{g}_{\mathrm{u}, \mathrm{s}}^{(\mathrm{i})}\right|^{2}}{\mathrm{~N}_{0} \frac{\mathrm{B}}{\mathrm{s}}}\right)$

It is equivalent to finding the maximum of the following cost function

$\mathrm{L}=\mathrm{u}=1+\lambda 1 \quad \mathrm{~s} \in \Omega \mathrm{uUs} \in \Omega \quad \log 21+\mathrm{pu}, \mathrm{sHu}, \mathrm{s}$ $\mathrm{uPtotu}=2 \mathrm{U} \lambda \mathrm{us} \in \Omega 1 \mathrm{~S} \log 21+\mathrm{p} 1, \mathrm{sH} 1, \mathrm{~s}-\alpha 1$ us $\in \Omega \mathrm{u} 1 \mathrm{Slog} 21$

$+\mathrm{pu}, \mathrm{sHu}, \mathrm{s}$

where $\left\{\lambda_{i}\right\}_{i=1}^{K}$ are the Lagrangian multipliers. This cost function is differentiated with respect to $p_{u, s}$ and set each derivative to 0 . The optimal power distribution for a single user can be obtained as

$\frac{\mathrm{H}_{\mathrm{u}, \mathrm{m}}}{1+\mathrm{H}_{\mathrm{u}, \mathrm{m}} \mathrm{p}_{\mathrm{u}, \mathrm{m}}}=\frac{\mathrm{H}_{\mathrm{u}, \mathrm{n}}}{1+\mathrm{H}_{\mathrm{u}, \mathrm{n}} \mathrm{p}_{\mathrm{u}, \mathrm{n}}}$

for $\mathrm{m}, \mathrm{n} \in \Omega_{\mathrm{u}}$ and $\mathrm{u}=1,2, \ldots, \mathrm{U}$. It is assumed that $\mathrm{H}_{\mathrm{u}, 1} \leq$ $\mathrm{H}_{\mathrm{u}, 2} \leq \cdots \leq \mathrm{H}_{\mathrm{u}, N_{u}}$ for $\mathrm{u}=1,2, \ldots, \mathrm{U}$ and $\mathrm{N}_{\mathrm{u}}$ is the number of subchannels in $\Omega_{\mathrm{u}}$ without the loss of generality. Thus, Equation 11 can be amended as

$\mathrm{p}_{\mathrm{u}, \mathrm{n}}=\mathrm{p}_{\mathrm{u}, 1}+\frac{\mathrm{H}_{\mathrm{u}, \mathrm{n}}-\mathrm{H}_{\mathrm{u}, 1}}{\mathrm{H}_{\mathrm{u}, \mathrm{n}} \mathrm{H}_{\mathrm{u}, 1}}$

for $n=1,2, \ldots, N_{u}$ and $u=1,2, \ldots, U$. This Equation 12 displays that the power distribution for a single user $u$ on subchannel n. By using Equation 12, $\mathrm{P}_{\mathrm{u} \text {,tot }}$ defined as the total power allocated for user ' $u$ ' can be illustrated as

$\mathrm{P}_{\mathrm{u}, \text { tot }}=\sum_{\mathrm{n}=1}^{\mathrm{N}_{\mathrm{u}}} \mathrm{p}_{\mathrm{u}, \mathrm{n}}=\mathrm{N}_{\mathrm{u}} \mathrm{p}_{\mathrm{u}, 1}+\sum_{\mathrm{n}=2}^{\mathrm{N}_{\mathrm{u}}} \frac{\mathrm{H}_{\mathrm{u}, \mathrm{n}}-\mathrm{H}_{\mathrm{u}, 1}}{\mathrm{H}_{\mathrm{u}, \mathrm{n}} \mathrm{H}_{\mathrm{u}, 1}}$

for $\mathrm{u}=1,2, \ldots, \mathrm{U}$. The water-filling algorithm will put more power into the subchannels with larger SNR in the frequency domain. The total power constraint and the capacity ratio constraint are used to take $\left\{\mathrm{P}_{\mathrm{u}, \text { tot }}\right\}_{\mathrm{u}=1}^{\mathrm{U}}$.

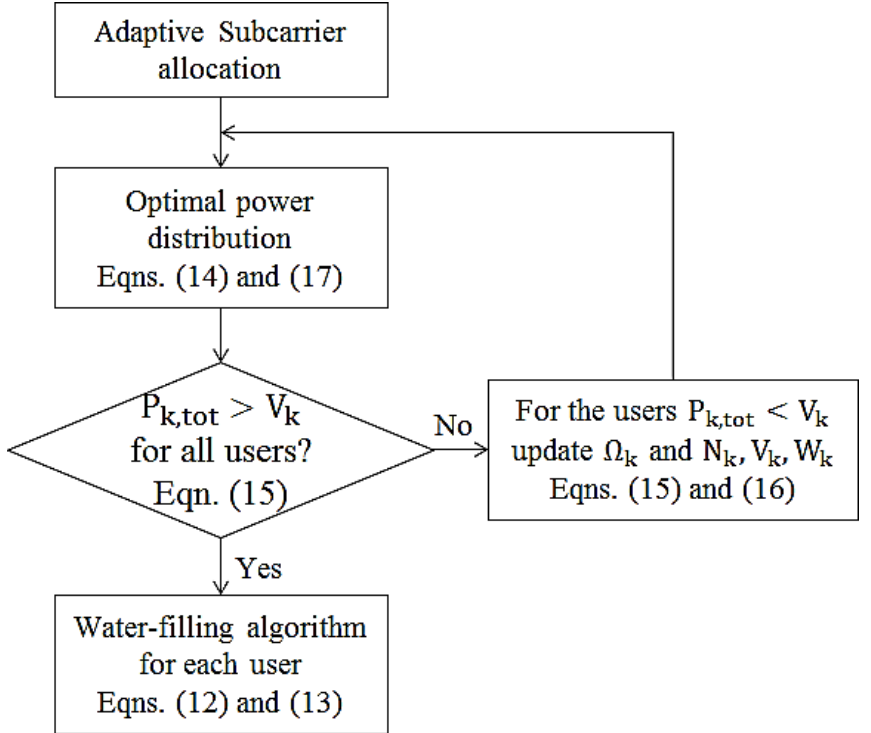

Fig -6: Adaptive power allocation based on allocated sub carriers

By means of Equation 11 and 13, the capacity ratio constraints can be stated as

$$
\begin{array}{r}
\frac{1}{\alpha_{1}} \frac{\mathrm{N}_{1}}{\mathrm{~N}}\left(\log _{2}\left(1+\mathrm{H}_{1,1} \frac{\mathrm{P}_{1, \text { tot }}-\mathrm{V}_{1}}{\mathrm{~N}_{1}}\right)+\log _{2} \mathrm{~W}_{1}\right)= \\
\frac{1}{\alpha_{\mathrm{u}}} \frac{\mathrm{N}_{\mathrm{u}}}{\mathrm{N}}\left(\log _{2}\left(1+\mathrm{H}_{\mathrm{u}, 1} \frac{\mathrm{P}_{\mathrm{u}, \text { tot }}-\mathrm{V}_{\mathrm{u}}}{\mathrm{N}_{\mathrm{u}}}\right)+\log _{2} \mathrm{~W}_{\mathrm{u}}\right)
\end{array}
$$

where $\mathrm{V}_{\mathrm{u}}$ and $\mathrm{W}_{\mathrm{u}}$ are said as

$\mathrm{V}_{\mathrm{u}}=\sum_{\mathrm{n}=2}^{\mathrm{N}_{\mathrm{u}}} \frac{\mathrm{H}_{\mathrm{u}, \mathrm{n}}-\mathrm{H}_{\mathrm{u}, 1}}{\mathrm{H}_{\mathrm{u}, \mathrm{n}} \mathrm{H}_{\mathrm{u}, 1}}$

And

$\mathrm{W}_{\mathrm{u}}=\left(\prod_{\mathrm{n}=2}^{\mathrm{N}_{\mathrm{u}}} \frac{\mathrm{H}_{\mathrm{u}, \mathrm{n}}}{\mathrm{H}_{\mathrm{u}, 1}}\right)^{\frac{1}{\mathrm{~N}_{\mathrm{u}}}}$

for $\mathrm{u}=1,2, \ldots, \mathrm{U}$.

The total power can be achieved by adding the total power constraints

$\mathrm{P}_{\mathrm{tot}}=\sum_{\mathrm{u}=1}^{\mathrm{U}} \mathrm{P}_{\mathrm{u}, \text { tot }}$

Solving the set of functions offers the optimal power allocation scheme. These equations are nonlinear. Optimal power distribution algorithm is shown in Figure 6.

\section{SIMULATION PARAMETERS AND RESULTS}

Simulation parameters and test results are described in Section 6. Table 1 shows the simulation parameters.

Table -1: Simulation parameters for the proposed system

\begin{tabular}{|l|l|}
\hline Number of Users & 4,16 \\
\hline Antenna Pair & $2 \times 4,4 \times 8$ \\
\hline Number of Frame & 10 \\
\hline
\end{tabular}




\begin{tabular}{|l|l|}
\hline Number of Subcarriers & 64,512 \\
\hline Carrier Frequency (fc) & $3.5 \mathrm{GHz}$ \\
\hline Bandwidth (BW) & $10 \mathrm{MHz}$ \\
\hline Total Power & $1 \mathrm{~W}$ \\
\hline Eb/N0 (dB) & $0,2,4,6,8,10$ \\
\hline Multipath & 6 \\
\hline Channel Estimation & Pilot-assisted \\
\hline
\end{tabular}

Figure 7 expresses the comparison of the sum capacity for three methods using 64 subcarriers and four users. When the methods are compared, adaptive resource allocation is better than static allocation.

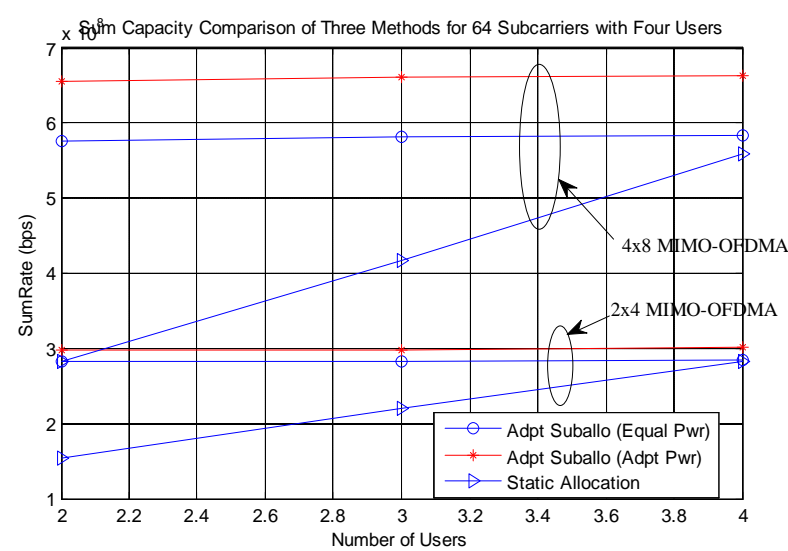

Fig -7: Comparison of sum capacity for three methods with 64 sub carries and four users

In adaptive allocation methods, allocating both the resources such as subcarriers and power adaptively can provide more capacity than allocating only subcarriers adaptively with equal power.

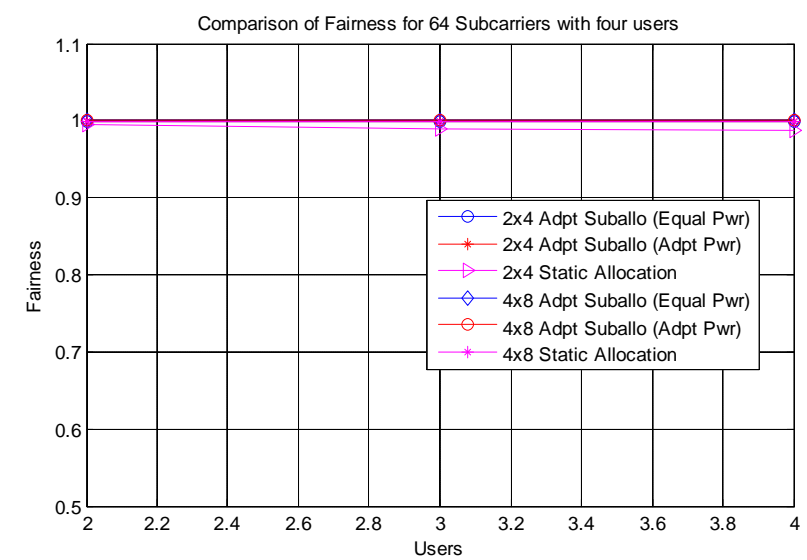

Fig -8: Comparison of fairness for three methods with 64 sub carries and four users

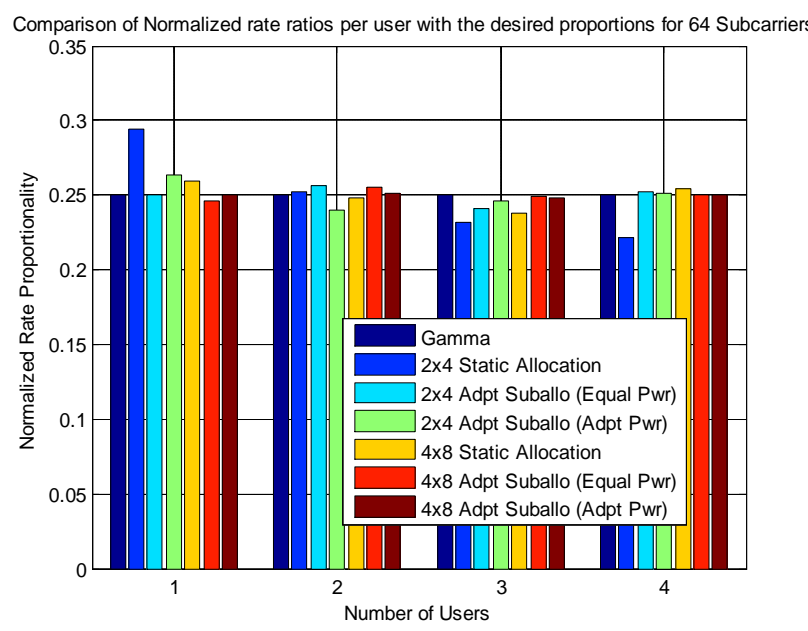

Fig -9: Comparison of normalized rate proportionality for three methods with 64 sub carries and four users

Comparing the antenna pairs, the increasing the number of antennas improves the capacity but increases the BER shown in Figure 13.

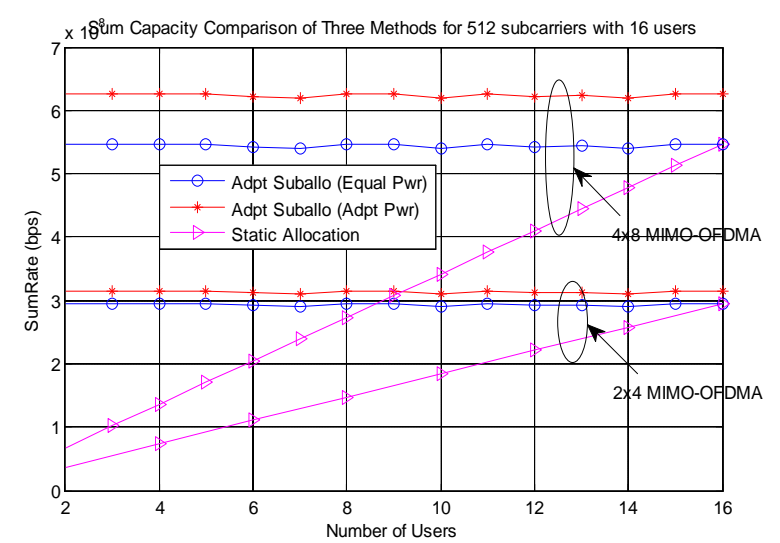

Fig -10: Comparison of sum capacity for three methods with 512 sub carries and sixteen users

Figure 8 shows the relation between the fairness and the users. The fairness range is between 0 and 1 . The most fairness is 1 .

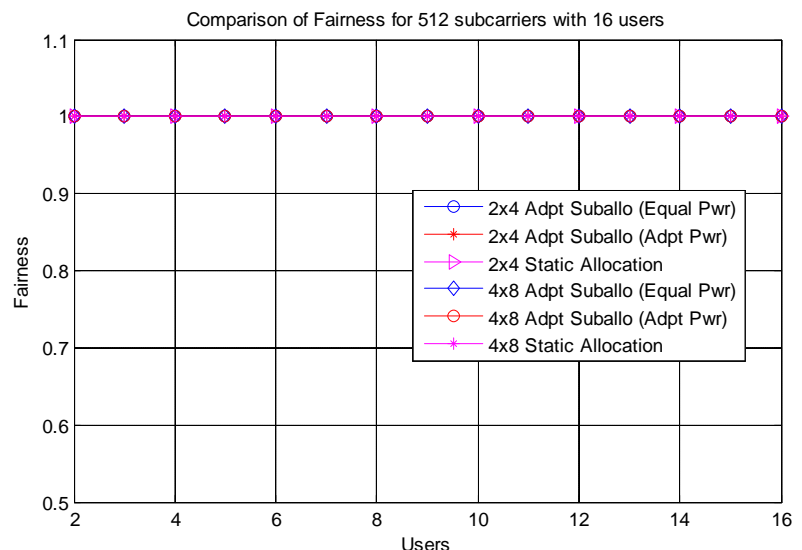

Fig -11: Comparison of fairness for three methods with 512 sub carries and sixteen users 
In this comparison, adaptive allocation can give more fairness than static allocation. The values of two adaptive allocation methods are not too different. Increasing the number of antennas can get more fairness.

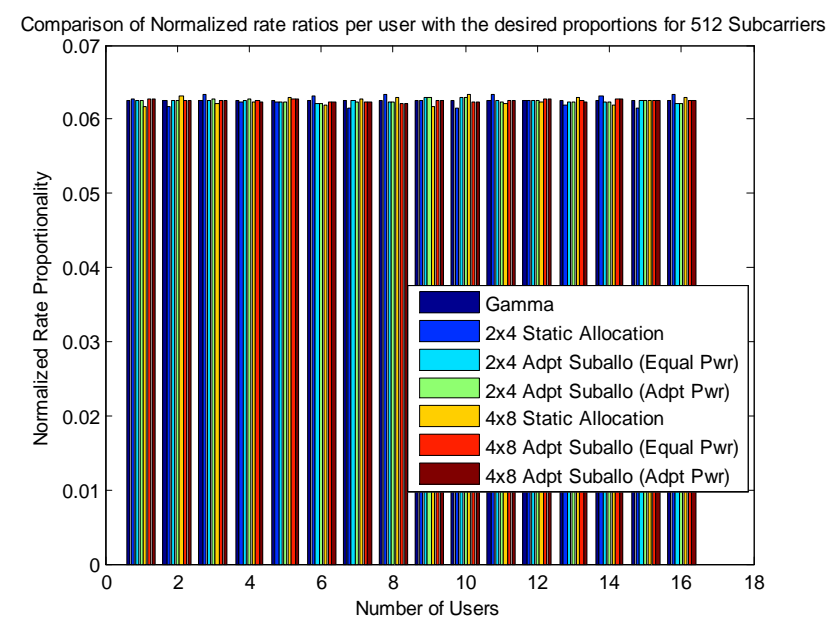

Fig -12: Comparison of normalized rate proportinality for three methods with 512 sub carries and sixteen users

Figure 9 shows that the rate is proportionality assigned to the users by using $R_{u} / \sum_{u=1}^{U} R_{u}$ where $U$ is the number of users. In these figures, the three methods are compared with 512 subcarriers and 16 users. It can be seen that increasing the number of subcarriers can give the most fairness than 64 subcarriers. In this system, it is assumed that the predetermined rate proportions are equal (1:1:1:1) for four users. Adaptive allocation gets nearly rate proportions with the desired proportion (Gamma). Therefore, the adaptive allocation can give more fairness. It can be found that $4 \times 8$ antenna configuration achieves the nearest rate proportion than $2 \times 4$ antenna configuration. Figure 10 to 12 shows the sum rate, fairness, and rate proportionality to the users respectively. Figure 13 compares the BER of 64 and 512 subcarriers for $2 \times 4$ and $4 \times 8$ antenna configurations.

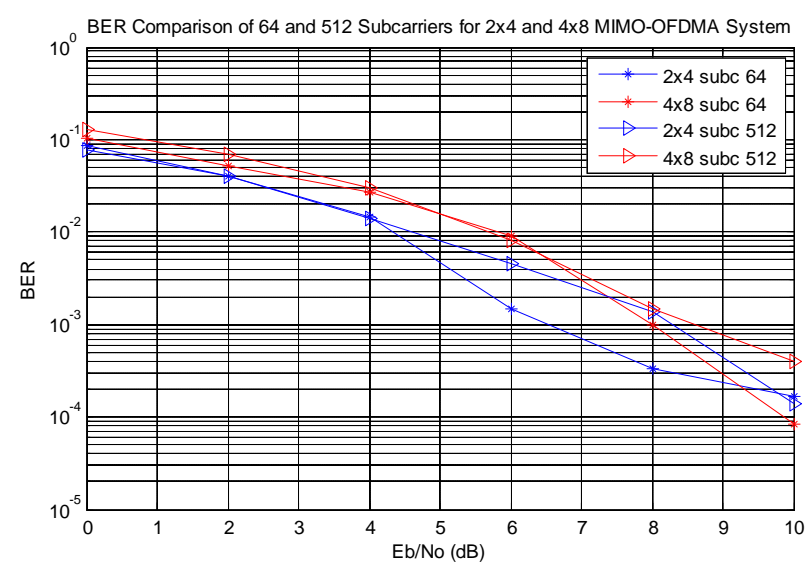

Fig -13: Comparison of BER for 64 and 512 subcarriers

It can be seen that the more subcarriers and the number of antennas increase the BER. Increasing the number of antennas improves not only the capacity but also the BER because the spatial multiplexing technique is used.

\section{CONCLUSIONS}

The proposed system aims to improve the spectrum efficiency. The spectrum efficiency can be expressed in terms of the system capacity. On the increasing capacity, it is considered to get the desired rate for each user. The fairness is also an important parameter. To consider a tradeoff between these two parameters (capacity and fairness), three methods are developed to compare the performance of the system. BER is the essential parameter to evaluate the system performance. To evaluate the performance of this system, 2x4 and 4x8 MIMO-OFDMA are chosen. In this system, the three methods such as static allocation (equal subcarrier allocation with equal power), adaptive subcarrier allocation with equal power, and adaptive subcarrier allocation with adaptive power distribution are compared. Adaptive allocation is better than static allocation for both capacity and fairness. Adaptive subcarrier allocation with adaptive power distribution increases the sum capacity than adaptive subcarrier allocation with equal power distribution shown in Figure 7 and 10. For these two methods, fairness is not too different. Therefore, adaptive subcarrier allocation with adaptive power distribution method is more efficient than adaptive subcarrier allocation with equal power distribution for the system capacity. Increasing the number of subcarriers can support the multiuser diversity and more fairness. Increasing the number of antennas improves the capacity and increases the BER. It can also be found that more antennas can give more fairness.

\section{ACKNOWLEDGEMENTS}

Firstly, I would like to special thanks to my parents for encouragement without any trouble throughout my life. Especially, I would like to express my sincere gratitude to supervisor Prof. Dr. Zaw Min Naing (Pro-Rector, Technological University Maubin, Myanmar) for his invaluable suggestions and appreciative guidance. And I would like to deeply thank to Co-supervisor Dr. Thiri Thandar Aung (Lecturer of Electronic Engineering Department) from Yangon Technological University, Myanmar, for her perfect guidelines for this paper. In addition, I really grateful to Dr. Thet Paing Phyo (Associate Professor of Electronic Engineering Department) from Yangon Technological University, Myanmar, for his appreciative comments and suggestions. I greatly express thanks to all persons whom will concern to support in preparing this paper for their technical support and guidance.

\section{REFERENCES}

MIMO - WIKIPEDIA, THE FREE ENCYCLOPEDIA.HTM.

[2] D.W.K. Ng, E.S. Lo, and R. Schober. Dynamic Resource Allocation in MIMO-OFDMA Systems with Full-Duplex and Hybrid Relaying, IEEE Transactions on Communications, 60(5):1291-1304, May 2012.

[3] Pratibha, Jagannatham, and Aditya K. Power allocation for MIMO-OFDM based CR with spatial constraints and CSI uncertainty. In SAM '12: 7th 
IEEE Sensor Array and Multichannel Signal Processing Workshop, pages 137-140, June 2012.

[4] T.F. Maciel and A. Klein On the Performance, Complexity and Fairness of Suboptimal Resource Allocation for Multi-User MIMO-OFDMA System, IEEE Transactions on Vehicular Technology, 59:406-419, Jan 2010.

[5] Bin Da, C. Ko, "A New Scheme with Controllable Capacity and Fairness for OFDMA Downlink Resource Allocation”, in Proc. IEEE VTC 2007-fall, pp. 1817-1821.

[6] J. Hui, and Y. Zhou, "Enhanced Rate Adaptive Resource Allocation Scheme in Downlink OFDMA System", in Proc. IEEE VTC2006-Spring, vol. 5, pp. 2464-2468.

[7] M. Kobayashi, G. Caire, "Iterative Water filling for Weighted Rate Sum Maximization in MIMO OFDM Broadcast Channels", in Proc. ICASSP Conf., vol. 3, April 2007, pp. 5-8.

[8] G. Li, and H. Liu, "On the Optimality of Downlink OFDMA MIMO Systems", in Proc. Signals, Systems and Computers Conf., vol. 1, November 2004, pp. 324-328.

[9] M.S. Maw, and I. Sasase, "Resource Allocation Scheme in MIMO -OFDMA System for users Different Data Throughput Requirements", in Proc. IEEE WCNC, March 2007, pp. 1706-1710.

[10] W. Rhee, and J. M. Cioffi, "Increase in Capacity of Multiuser OFDM System Using Dynamic Subchannel Allocation", in Proc. IEEE VTC, Japan, May 2000, pp. 1085-1089.

[11] Z. Shen, J. G. Andrews, and B. L. Evans, "Adaptive Resource Allocation in Multiuser OFDM Systems with Proportional Rate Constraints", IEEE Trans. wireless communication, vol. 4, pp. 2726 - 2737, November 2005.

[12] I. C. Wong, ZukangShen, B. L. Evans, and J. G. Andrews, A Low Complexity algorithm for Proportional Resource Allocation in OFDMA Systems ${ }^{\text {ee }}$, in Proc. Signal Processing Systems Conf., SIPS 2004, pp. 1-6.

[13] J. Xu, J. Kim, W. Paik, J. S. Seo, „Adaptive Resource Allocation Algorithm with Fairness for MIMO-OFDMA System ${ }^{e}$, in Proc.VTC 2006spring, vol. 4, pp. 1585-1589.

[14] J. M. Choi, J.S. Kwak, H. S. Kim,and J. H. Lee, "Adaptive Subcarrier Bit, and Power Allocation Algorithm for MIMO-OFDMA System", in Proc. VTC 2004-Spring, vol.3, pp.1801-1805.

[15] C. Y. Wong, R. S. Cheng, K. B. Letaief, and R. D. Murch, Multiuser OFDM with Adaptive Subcarrier, Bit, and Power Allocation "e, IEEE J. Select. Areas Communication, vol. 17, pp. 1747- 1758, October 1999.

[16] Y. J. Zhang, and K. B. Letaief, „An Efficient Resource-Allocation Scheme for Spatial Multiuser Access in MIMO/OFDM Systemse, IEEE Trans Commun., vol.53, pp.107-116, January 2005.

[17] H. Rohling and R. Gruenheid, Performance comparison of different multiple access schemes for the downlink of an OFDM communication system, in Proceedings of the $47^{\text {th }}$ IEEE Vehicular Technology Conference (VTC '97), pp. 1365-1369, Phoenix, Fla, USA, May 1997.

[18] Y.S. Cho, J. Kim, W.Y. Yang, and C.G. Kang, "MIMO-OFDM Wireless Communications with MATLAB”, ISBN 978-0-470-82561-7.

\section{BIOGRAPHIES}

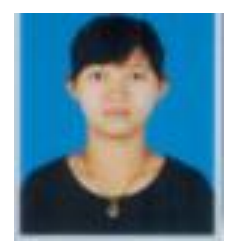

The author received the Bachelor and Master degree of Information Technology at the Department of Information Technology from Pathein Technological University in 2008 and 2011. She is currently a $\mathrm{PhD}$ student of Electronic Engineering at the Department of Electronic Engineering at Yangon Technological University, Myanmar. Her research interests include multicarrier communication systems, multiple input multiple output technology, resource allocation in multiuser environments.

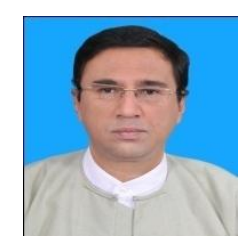

The author is currently a Pro-Rector of Technological University (Maubin) under Department of Technical and Vocational Education, Ministry of Science and Technology (MOST), the Republic of the Union of MYANMAR. He received his B.E degree from Yangon Technological University (YTU) in 1992, M.E degree from YTU in 1999, and Ph.D from the School of Electrical and Electronics Engineering, Nanyang Technological University, Singapore in 2005 all in electronics and communication engineering. He has vast experience as an engineer, academia and researcher in the field of electronics control, satellite communication, modeling and simulation of RADAR and unmanned aerial vehicle (UAV), performance analysis of network resource management, wireless/mobile communications and networking, switching and routing, ad-hoc networks. He is the editor of the International Journal of Ultra Wideband Communications and Systems published by Indian Institute of Technology (Guwahait), India and the editor for the journal of Ministry of Science and Technology, Myanmar. Moreover, $\mathrm{He}$ is also the editorial board member ASEAN Journal Science and Technology Development (AJSTD).

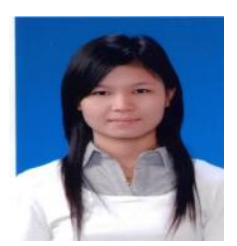

The author was awarded a Bachelor and Master degree of Electronic Engineering at the Department of Electronic Engineering, Mandalay Technological University and Yangon Technological University in 2004 and 2006. She finished her Ph. D. degree at the Department of Electrical and Electronic Engineering, Faculty of Engineering, Chulalongkorn University in 2014. She is now working as a Lecturer in Yangon Technological University since 2012. 
The author received the Bachelor degree of Electronic Engineering at the Department of Electronic Engineering from Mandalay Technological University in 2004. He was achieved the Master and $\mathrm{Ph}$. D. degree of Electronic Engineering at the Department of Electronic Engineering from Yangon Technological University and Mandalay Technological University in 2006 and 2009. He was awarded the Post Graduate Diploma (ISRO, India) in 2012. He served as a lecturer at the office of Ministry of Science and Technology, Nay Pyi Taw. He is currently working as an Associate Professor at the Department of Electronic Engineering, Yangon Technological University. 